\title{
The B-cell maturation factor Blimp-1 specifies vertebrate slow-twitch muscle fiber identity in response to Hedgehog signaling
}

\author{
Sarah Baxendale ${ }^{1}$, Claire Davison ${ }^{1}$, Claire Muxworthy ${ }^{1}$, Christian Wolff ${ }^{1}$, Philip W Ingham $^{1}$ \& Sudipto Roy ${ }^{1,2}$
}

Vertebrate skeletal muscles comprise distinct fiber types that differ in their morphology, contractile function, mitochondrial content and metabolic properties. Recent studies identified the transcriptional coactivator PGC $-1 \alpha$ as a key mediator of the physiological stimuli that modulate fiber-type plasticity in postembryonic development ${ }^{1}$. Although myoblasts become fated to differentiate into distinct kinds of fibers early in development, the identities of regulatory proteins that determine embryonic fiber-type specification are still obscure. Here we show that the gene u-boot (ubo), a mutation in which disrupts the induction of embryonic slow-twitch fibers ${ }^{2}$, encodes the zebrafish homolog of Blimp-1, a SET domain-containing transcription factor that promotes the terminal differentiation of $B$ lymphocytes in mammals ${ }^{3}$. Expression of $u b o$ is induced by Hedgehog $(\mathrm{Hh})$ signaling in prospective slow muscle precursors, and its activity alone is sufficient to direct slowtwitch fiber-specific development by naive myoblasts. Our data provide the first molecular insight into the mechanism by which a specific group of muscle precursors is driven along a distinct pathway of fiber-type differentiation in response to positional cues in the vertebrate embryo.

In the developing somites of the zebrafish embryo, mononucleate muscle fibers of the slow-twitch lineage derive from the adaxial cells, a distinct subpopulation of the paraxial mesoderm that flanks the notochord and is the first to enter myogenesis ${ }^{2,4}$. This early myogenic commitment and allocation to the slow-twitch lineage is directed by members of the Hh family of secreted glycoproteins that are expressed by axial midline tissues ${ }^{5-7}$. Loss of Hh signaling, caused by mutational inactivation of genes encoding Sonic hedgehog (Shh) or the Hhtransducing protein Smoothened (Smo), impairs the development of some or all cells of the slow muscle lineage ${ }^{8,9}$, as shown by the loss of expression of the slow myosin heavy chain $(\mathrm{MyHC})$ isoform and the homeodomain protein Prox $1-$ markers that are exclusively expressed by slow-twitch fibers ${ }^{2,10}$. Conversely, ectopic Hh activity is sufficient to transform other cells of the paraxial mesoderm, which are otherwise fated to differentiate as syncytial fast-twitch fibers, to the slow muscle fate $e^{5-7,11}$.

Embryos homozygous with respect to a mutation at the $u b o$ locus also show a loss of slow-twitch fibers, similar to that seen in embryos lacking Smo activity ${ }^{2}$. In contrast to the latter, however, other aspects of the developing embryo that depend on Hh signaling are unaffected in $u b o$ mutant embryos. In addition, whereas $u b o$ activity is required cell-autonomously for the acquisition of slow-twitch fiber identity ${ }^{2}$, myotomal cells in $u b o$ mutants retain the competence to transduce $\mathrm{Hh}$, as judged by their activation of the Hh target genes patched 1 ( $p t c 1)$ and myoD (see below). Thus, it is the ability of the muscle precursors to adopt the slow-twitch fiber fate in response to $\mathrm{Hh}$, rather than the generation or transduction of the signal itself, that is specifically compromised by the $u b o$ mutation. On the basis of these observations, we previously proposed that $u b o$ acts as a Hh-regulated switch that selects the pathway of slow-twitch fiber differentiation in cells committed to the myogenic lineage ${ }^{2}$.

To determine the molecular nature of this switch, we adopted a positional cloning strategy to isolate $u b o$ (Fig. 1a). By analyzing the products of 3,060 meioses, we mapped $u b o$ to a region located $0.42 \mathrm{cM}$ from the marker $z 6329$ on linkage group 16 (LG16). We injected PAC and BAC clones spanning the candidate region into $u b o$ mutant embryos and assayed their ability to rescue the development of slowtwitch fibers by using the expression of Prox 1 as a marker. One such PAC clone, BUSMP706F2291, restored myotomal expression of Prox1 (Fig. 1b-d and Supplementary Table 1 online), indicating that the candidate gene mutated in $u b o$, together with the regulatory elements required for its appropriate spatiotemporal expression in the precursors of the slow lineage, are located on this genomic clone. Sequence analysis of the critical genomic interval identified only one transcription unit, encoding a protein with substantial similarity to the mammalian SET domain-containing zinc-finger transcription factor Blimp-1 (ref. 3; Fig. 1f), making it the sole candidate for the gene inactivated by the $u b o$ mutation.

${ }^{1}$ MRC Intercellular Signaling Group, Center for Developmental Genetics, School of Medicine and Biomedical Sciences, University of Sheffield, Firth Court, Western Bank, Sheffield S10 2TN, UK. ${ }^{2}$ Institute of Molecular and Cell Biology, 30 Medical Drive, 117609 Singapore. Correspondence should be addressed to P.W.I. (p.w.ingham@sheffield.ac.uk).

Published online 21 December 2003; doi:10.1038/ng1280 
Using PCR, we assembled a full-length cDNA clone corresponding to zebrafish $u b o$ and analyzed its expression pattern during embryonic development by whole-mount in situ hybridization. Expression of $u b o$ initiates during early gastrulation in the yolk syncytial layer and continues in cells of the involuting mesendoderm around the leading edge of the embryo (Fig. 2a,b). Expression is also prominent in the prechordal mesoderm throughout this period, culminating in high levels during early stages of somitogenesis (Fig. 2a,c). In the somitic mesoderm, expression is first apparent around the tail-bud stage and is confined exclusively to the adaxial cells from the outset (Fig. 2d). As development proceedes, expression remains restricted to the adaxial cells but becomes more pronounced, with very high levels detectable at the three-somite stage (Fig. 2e). Adaxial expression then begins to diminish and, before the characteristic radial 'migration' of the slowtwitch fibers ${ }^{4}$ occurs, decays rapidly in a rostro-caudal progression (Fig. 2f,g), such that by $24 \mathrm{~h}$ post fertilization (h.p.f.) remnants of the pattern are evident only in the terminal tail somites (Fig. 2h). Thus, expression of $u b o$ initiates in the adaxial cells after the onset of expression of $m y o D^{12}$ and myf5 (ref. 13), which is indicative of the cells' commitment to the myogenic lineage, but before the expression of slow MyHC and Prox1 (ref. 2), which marks the onset of the cells' terminal differentiation into slow-twitch fibers.

To confirm that the disruption of slowtwitch fibers is caused by mutation of $u b o$, we amplified and sequenced $u b o$ cDNA clones from embryos homozygous with respect to

Figure 1 Positional cloning of the ubo locus. (a) Genetic map of the ubo locus on LG16, showing the relative positions of the simple sequence-length polymorphism ( $\mathrm{z}$ ) markers and the mapped expressed-sequence tags on the LN54 and T51 radiation hybrids. The expanded critical genomic region shows the number of recombinants per 3,060 meioses obtained with each marker. The positions of the PAC F2291 and the sequenced clone zK11F5 are shown, along with those of the known markers (red) and the new genetic markers (blue diamonds). Two transcripts identified by the sequence annotation of zK11F5 are indicated (blue arrows).

(b) Expression of Prox 1 in the nuclei of the slow-twitch fibers in the myotome of a wild-type embryo. (c) Complete absence of Prox 1 expression in the myotome of an embryo homozygous with respect to the $u b o^{\text {tp39 }}$ allele. (d) An ubo mutant embryo injected with PAC F2291, showing restoration of Prox1 expression in the slow-twitch fibers. (e) An ubo mutant embryo, showing restoration of Prox1-expressing slow-twitch fibers on misexpression of $u b o$ from the hs-blimp1-IRES-gfp construct. In $\mathbf{b}-\mathbf{e}$, embryos are at 24 h.p.f. and oriented laterally with anterior to the left and dorsal to the top. (f) Amino acid sequence alignment of the Blimp1 homologs of zebrafish, mouse and human. Identical residues are shown in red, conserved residues in blue. The zinc-fingers (underlined), the SET domain (boxed) and the positions of the missense mutations in the $u b o^{\text {tp } 39}$ allele (asterisks) and the splice site-specific morpholino oligonucleotide (arrow) are indicated. the only extant mutated allele, $u b o^{\mathrm{tp} 39}$. This identified two potential missense mutations: S481F in the region between the SET domain and the first zinc finger, and H564R in the second zinc finger (Fig. 1f). Such amino acid substitutions raise the possibility that $u b o^{\mathrm{tp} 39}$ represents a hypomorphic rather than an amorphic allele of $u b o$. This would be consistent with our previous observations that prospective slow muscle precursors of $u b o$ mutant embryos retain the capacity to initiate, albeit transiently, the earliest events of slow-twitch fiber differentiation in response to Hh signaling, before aberrantly differentiating into fast muscle cells ${ }^{2}$.

To investigate the effects of complete loss of $u b o$ activity, we injected wild-type embryos with an antisense morpholino oligonucleotide designed to disrupt efficient splicing of its nascent $\mathrm{mRNA}^{14}$. In contrast to $u b o$ mutants, the $u b o$ morphants generated with a high dose of morpholino oligonucleotide showed early developmental defects manifested in considerable abbreviation of their head primordium a
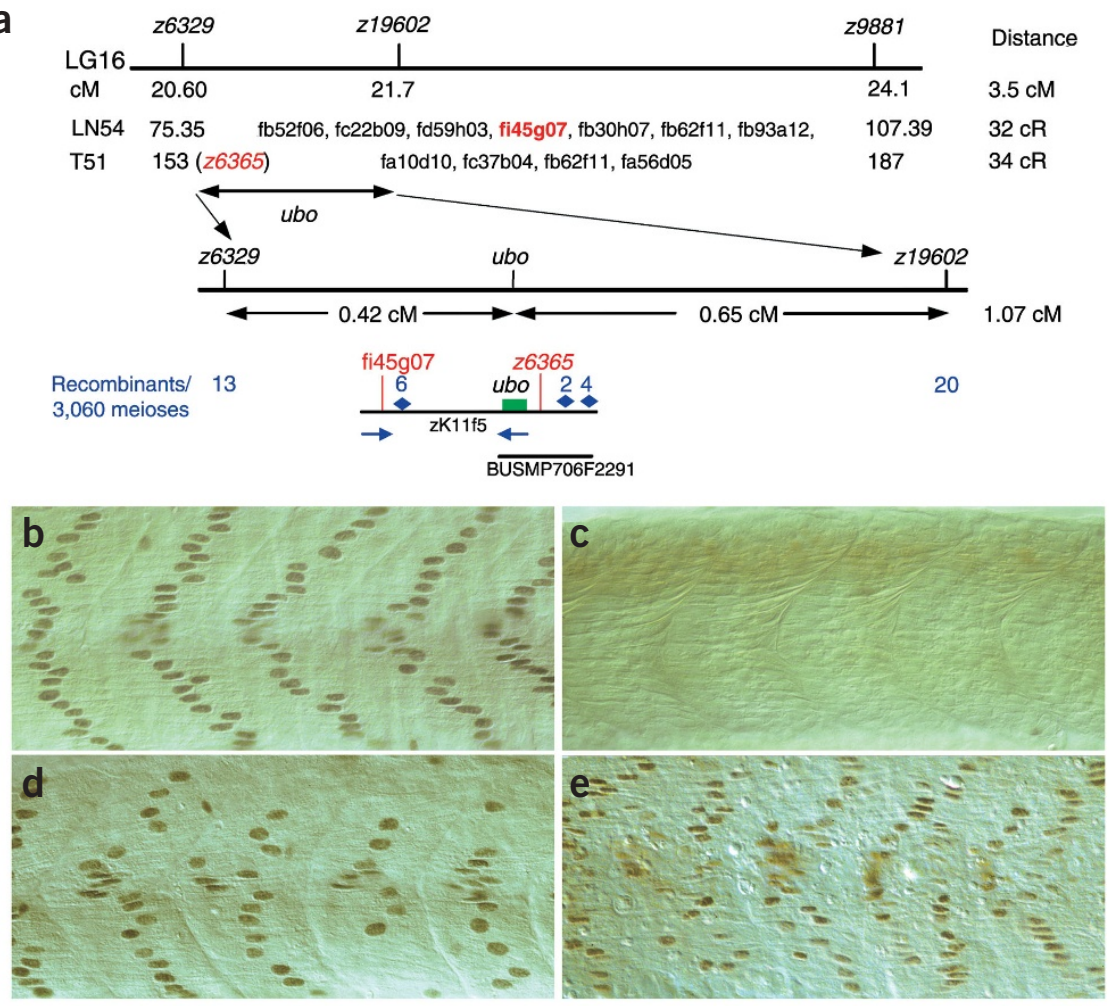

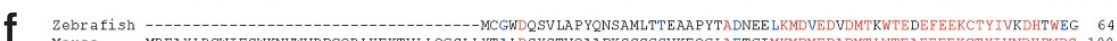
Mouse MREAY LRCWI F SWKNVWVRPCQR LHF KTVLLQGSLLYTA LDS YSTVQAAPKSSSGSVKF QGLAETGIMKMDMEDADMT LWTEAEF EEKCT YI VNDH PWDS 100

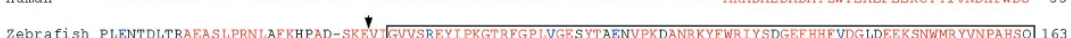

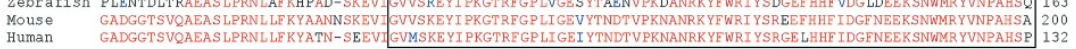

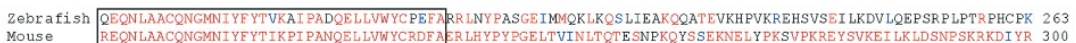

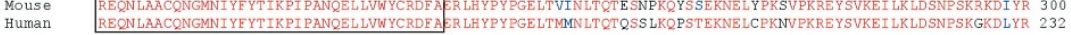
Zebrafish S---

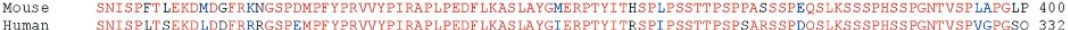
zebrafish EPREAF LPF SPALYNRGLNHY PGYSPAGSMPF YPNPHYSR YLMHHYPVSS LSGPST LGGIF PHMYPFYSSLVP PHVPFPP SMLSSEGSRQFMLPPDSPA- 426

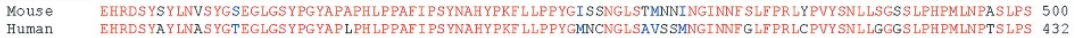

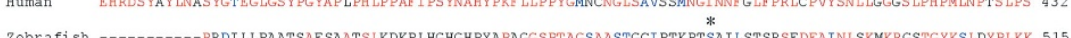
Zebrafish --1.---PRDI LLPAATSAF SAATS LKDKP LHGHGHPYAPAGGSPTAGSAASTGCI PTKPTSAI LSTSRSEDEAINLSKMKRGSTGYKS LDYPLKK 515

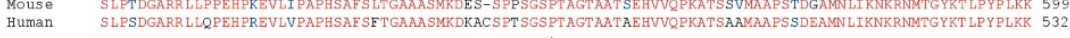
Zebra fish QNGKI KYECNVCTKTFGQLSNLKVH LRVHSGERPFKCQTCNKGFTQLAH * LRHY LVHTGERPHECQVCHKRF SSTSNLKTHLRLHSGEKP YQCRICPAKF 615 Mouse
Human Zebrafish TQFVHLK LHKRLHTRER PHQCPHCHHNYIHLSS LRFH LKGYC LAVSPSPSCS LDE LNRVNEEIEREDISDNADR LEEME-GF DVEGMVERQIFGLLWQEM 714

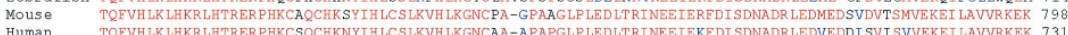

Zebrafish DFKASYHKGSTGGD LHPRAPA LSAYH LNEHDSEASVIKVHRSSPIQLLPIKVKKETEEAMDT 776

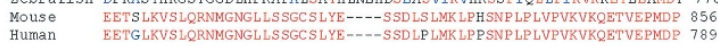


and shortening of the body axis (data not shown), consistent with a previously described role of Blimp-1 in regulating vertebrate gastrulation $^{15}$. Examination of the slow muscle lineage in these morphants at early stages of somitogenesis showed that its development was completely impaired, as indicated by the total absence of slow $\mathrm{MyHC}$; this contrasts with $u b o^{\mathrm{tp} 39}$ mutants, in which we detected variable expression of slow MyHC (Fig. 3a-c).

Despite this arrest, the adaxial cells retained their ability to transduce $\mathrm{Hh}$ and to commit to the myogenic fate, as indicated by the normal expression patterns of $p t c 1$ and $m y o D$ (Fig. 3d,e and data not shown), endorsing the view that $u b o$ is required to activate the program of slow-twitch fiber-specific development only after the decision to enter myogenesis has been made. A lower dose of morpholino oligonucleotide produced embryos with a phenotype reminiscent of that of $u b o$ mutants, with characteristic U-shaped somites devoid of Prox1 expression (Fig. 3f, compare with Fig. 1b,c) but with sporadic occurrence of slow MyHC-expressing muscle fibers irregularly distributed in the myotome (Fig. $\mathbf{3 g}-\mathbf{i}$ ). This indicates that at submaximal concentrations the morpholino oligonucleotide effectively phenocopies the $u b o^{\mathrm{tp} 39}$ allele.

Because Hh signaling is both necessary and sufficient for specification of slow-twitch fiber identity, we investigated whether Hh activity induces expression of $u b o$ in the adaxial cells. In slow-muscle-omitted (smu) embryos that lack Smo activity and thus cannot transduce $\mathrm{Hh}^{9}$, the expression of $u b o$ was specifically eliminated from the adaxial cells (Fig. 4a, compare with Fig. 2f). Furthermore, the inability of PAC F2291 to restore slow muscle development when injected into smu mutants was consistent with a requirement for Hh signaling to activate $u b o$ transcription in the slow muscle precursors (data not shown). Conversely, ubiquitous activation of the $\mathrm{Hh}$ pathway resulted in the ectopic expression of $u b o$, but only in the paraxial mesoderm (Fig. 4b, compare with Fig. 2f). Such limited competence of cells to activate $u b o$ expression in response to Shh suggests that core myogenic regulatory factors, such as Myf5 or MyoD, may act in concert with the Hh-regulated Gli proteins ${ }^{16}$ to activate the $u b o$ promoter.

To determine whether $u b o$ is sufficient to drive cells down the pathway of slow-twitch fiber differentiation, we investigated the effects of its misexpression on the specification of muscle fiber identity. Transient misexpression of $u b o$ driven by a heat-shock promoter not only restored slow muscle development in $u b o$ mutant embryos (Fig. 1e and Supplementary Table 1 online), but also induced supernumerary slow-twitch fibers in the myotome of wild-type embryos (Fig. 4c,d and Supplementary Table 1 online).

As a more stringent test of this ability of $u b o$ to direct slow-twitch myogenesis, we analyzed the result of its misexpression in smu mutant embryos, where, in the absence of Hh signaling (and the concomitant lack of $u b o$ expression), slow muscle development is arrested completely and all myotomal cells enter the default pathway of fast fiber-specific development ${ }^{9}$. Transient expression of $u b o$ in the muscle precursors of such mutants was sufficient to reprogram their fate to that of the slow-twitch identity, as judged by the presence of mononucleated fibers expressing slow MyHC and Proxl (Fig. 4e,f and Supplementary Table 1 online). By contrast, misexpression of the $u b o^{\mathrm{tp} 39}$ mutated cDNA in smu mutants had no such effect on muscle cell identity (Fig. $\mathbf{4 g}$ and Supplementary Table 1 online). Thus, ubo activity is both necessary and sufficient to initiate the slow-twitch developmental program in cells committed to the myogenic fate and, accordingly, constitutes a Hh-regulated molecular switch that controls this process.

Using the same assay, we investigated which of the two amino acid substitutions associated with the $u b o^{\text {tp } 39}$ allele is responsible for the loss of wild-type activity by introducing each of the nucleotide changes into the wild-type cDNA. Whereas expression of the S481F construct, like wild-type $u b o$, fully rescued slow-twitch fiber formation in smu embryos (Fig. $4 \mathrm{~h}$ and Supplementary Table $\mathbf{1}$ online), expression of the H564R construct showed a marked reduction in the
Figure 2 Expression pattern of ubo during embryonic development. (a) Dorsal view of a whole-mount wild-type embryo at $70 \%$ epiboly, showing expression in the prechordal mesoderm (long arrow) and cells of the YSL (short arrows). (b) The same embryo, oriented with dorsal to the top, showing expression in the mesendodermal cells along the involuting margin surrounding the yolk (arrows). (c) Ventral view of an embryo at the 2-3-somite stage, showing high expression in the prechordal mesoderm (arrows) (d) Dorsoventrally flattened preparation of a tail bud-stage embryo, showing onset of expression in the adaxial cells (arrows). (e) Similar preparation of an embryo at the 2-3-somite stage, with prominent expression in the adaxial cells (arrows) flanking the notochord. (f) Dorsal view of an embryo at the 12-somite stage, with expression receding in the anterior-most somites (small arrow) but remaining high in the newly formed posterior somites and segmental plate (long arrow). (g) Lateral view of the myotome of an embryo at the 18-somite stage. ubo expression at this stage has largely disappeared from the anterior somites (small arrow) and is restricted to the slow muscle precursors in the posterior somites (long arrow). (h) At 24 h.p.f., myotomal expression of $u b o$ is detectable only in the terminal tail somites (arrow). In $\mathbf{d}-\mathbf{h}$, anterior is to the left. a

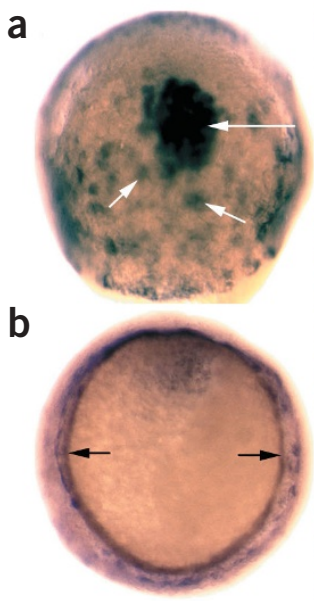

C

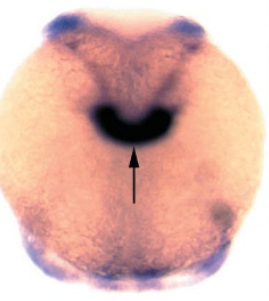

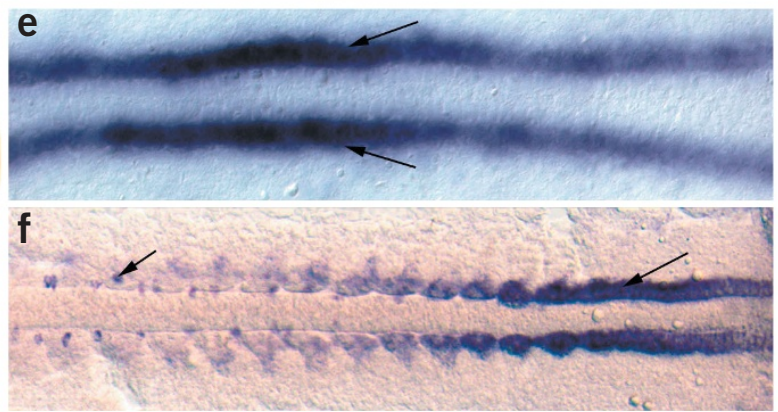
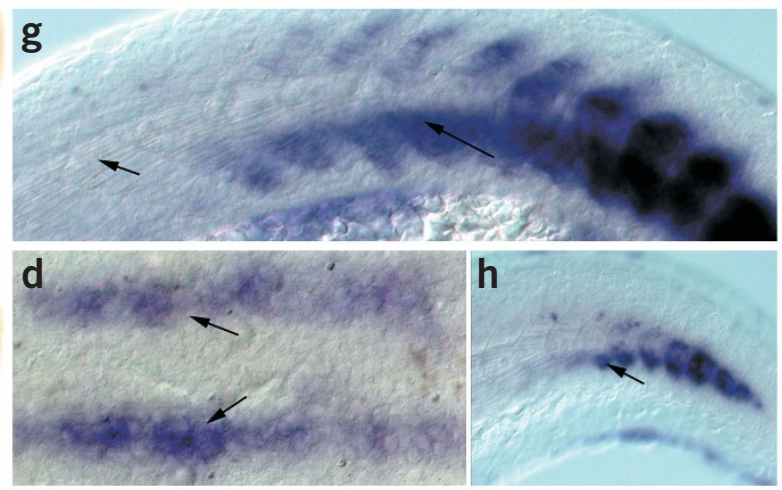
induction of slow-twitch fibers (Fig. 4i and Supplementary Table 1 online). This suggests that S481F represents a polymorphism in the genetic background that has no detrimental consequence to the activity of the protein, whereas H564R, in combination with S481F, severely compromises the ability of Ubo to function in slow-twitch fiber specification and is responsible for the $u b o$ mutant phenotype. Consistent with this observation, the first and second zinc fingers of mammalian Blimp-1 are essential for its sequence-specific DNA-binding activity ${ }^{17}$.

So far, the most detailed studies of Blimp-1 have focused on its role in B-lymphocyte maturation in mammals. Expression of Blimp-1 accompanies the differentiation of B cells into plasma cells, and its ectopic expression in cultured BCL1 cells is sufficient to induce such differentiation $^{3}$. In this context, Blimp-1 seems to act exclusively as a transcriptional repressor. The SET domain is thought to confer histone lysine methyltransferase activity on the protein ${ }^{18}$ and has been shown to repress transcription of the genes $M Y C^{19}$, IFNB1 (ref. 20) and $M H C 2 T A^{21}$ by binding to their promoters and recruiting the corepressors Groucho ${ }^{22}$ and histone deacetylase $\mathrm{e}^{23}$.

Our analysis has identified an unexpected and separate role of Blimp-1 in directing the differentiation of the embryonic slow muscle lineage in response to $\mathrm{Hh}$ signaling. In this context, $u b o$ activity is necessary not only for the repression of genes encoding fast lineage-specific markers, such as fast $\mathrm{MyHC}$, but also for the activation of genes encoding the slow MyHC isoform and Prox 1 proteins ${ }^{2}$. Possession of a SET domain is not inconsistent with Blimp-1 acting in transcriptional activation, because the methylation of some histone $\mathrm{H} 3$ lysine residues is associated with transcriptional activation ${ }^{18}$. At least in B cells, however, Blimp-1 is known to induce the transcription of some genes by repressing the expression of another transcriptional repressor, Pax5 (ref. 24).

Although our report provides the first evidence that Blimp-1 is involved in regulating the specification of embryonic types of muscle fiber, we note that previous studies have documented its expression in the developing myotome of the mouse $\mathrm{e}^{25}$. Because Hh signaling organizes somitic myogenesis in amniotes ${ }^{26}$, as well as in teleosts, this implies that Blimp-1 has an evolutionary conserved role in vertebrate muscle development and may shed light on the origin and diversification of different muscle lineages in the vertebrate embryo.

\section{METHODS}

Zebrafish strains and genetics. We maintained the $u b o^{\mathrm{tp} 39}$ mutation, which was isolated in a large-scale chemical mutagenesis screen at the Max-Planck Institut für Entwicklungsbiologie ${ }^{27}$, in the $\mathrm{AB}$ genetic background and outcrossed it to the polymorphic WIK(L11) strain of wild-type fish to generate mapping embryos for positional cloning. Fish carrying the $s m u^{\mathrm{b} 641}$ mutation were a gift from S. Devoto (Wesleyan University, Connecticut, USA).

Positional cloning of the $\boldsymbol{u b o}$ locus. $u b o$ has been mapped to LG16 (R. Geisler, personal communication). We screened a collection of simple sequence-length polymorphism markers positioned on the MGH linkage map as described ${ }^{28}$ and narrowed the position of $u b o$ to a 1.1-cM interval between the markers $z 6329$ and $z 19602$ (Fig. 1a). We used flanking markers and expressed-sequence tags that map to this interval on the L54 and T51 radiation hybrid maps (Zebrafish Information Network) to isolate PAC and BAC clones. We screened genomic libraries by hybridizing ${ }^{32} \mathrm{P}$-labeled DNA fragments to arrayed filters (C. Amemiya PAC library, RZPD and CHORI-211 BAC library; BACPAC Resources) or by PCR of pooled clones (C. Amemiya PAC library, RZPD) using
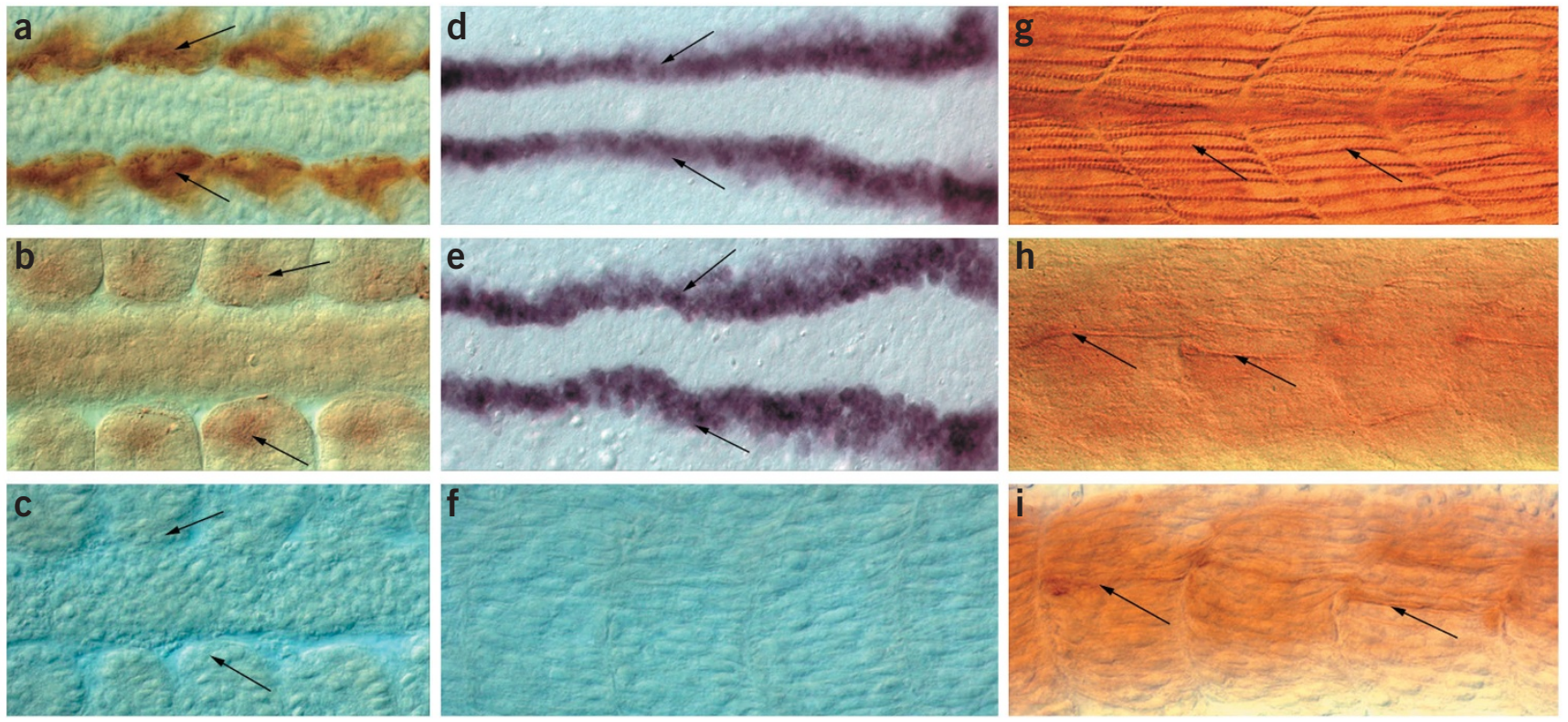

Figure 3 Dose-dependent effect of ubo morpholino oligonucleotide on slow-twitch fiber specification. (a) Expression of slow MyHC in the adaxial cells (arrows) of a wild-type embryo at the 10-somite stage. (b) An ubo mutant embryo at the 10-somite stage, showing reduced amounts of slow MyHC in the adaxial cells (arrows). (c) Complete absence of slow MyHC in the adaxial cells (arrows) of a ubo morphant embryo at the 10-somite stage injected with a high dose of morpholino oligonucleotide. (d) Expression of $m y o D$ at the 2-3-somite stage in the adaxial cells (arrows) of an embryo derived from a cross of $u b 0^{\text {tp } 39}$ heterozygous fish. The expression pattern in the mutants is indistinguishable from that in their wild-type siblings. (e) Wild-type expression of myoD in the adaxial cells (arrows) of a ubo morphant embryo at the 2-3-somite stage injected with a high dose of morpholino oligonucleotide. The axial midline appears thicker than in the wild-type control owing to improper gastrulation. (f) Absence of Prox1 expression in the myotome of a 24-h.p.f. ubo morphant embryo injected with a lower dose of morpholino oligonucleotide. Note the U-shaped morphology of the somites. (g) Expression of slow MyHC in the mature slowtwitch fibers (arrows) of a wild-type embryo at 24 h.p.f. (h) Small amounts of slow MyHC are detectable in a few fibers aberrantly distributed in the myotome (arrows) of a ubo mutant embryo at 24 h.p.f. (i) Similar pattern of slow MyHC expression in the myotome (arrows) of a ubo morphant injected with a low dose of morpholino oligonucleotide. Dorsal views are shown in a-e, lateral views in $\mathbf{f}-\mathbf{i}$. In all panels, embryos are oriented with anterior to the left. 
Figure 4 Hh signaling induces ubo expression in the adaxial cells and this activity is sufficient to specify slow-twitch fibers. (a) A smu mutant embryo at the 12-somite stage, showing complete absence of $u b o$ expression in the adaxial cells (arrows). (b) A wild-type embryo at the 12-somite stage injected with shh mRNA, showing activation of $u b o$ transcription throughout the paraxial mesoderm (arrows). (c) A wild-type embryo at 24 h.p.f., showing expression of slow MyHC (blue) and Prox1 (red) in the mature slow-twitch fibers. (d) A 24-h.p.f. wild-type embryo injected with hs-blimp1IRES-gfp, showing supernumerary slow-twitch fibers on misexpression of $u b o$. Slow fibers coexpressing GFP (green) from the hs-blimp1IRES-gfp construct are indicated (arrows). (e) Complete absence of slow MyHC and Prox 1 expression in the myotome of a smu mutant embryo at 24 h.p.f. (f) Induction of slow-twitch fibers expressing slow MyHC and Prox 1 in a smu mutant embryo on ectopic expression of the wild-type ubo cDNA. Slow fibers coexpressing GFP (green) are indicated (arrows). (g) Absence of slow-twitch fibers in a smu embryo on ectopic expression of the $u b o^{\text {tp} 39}$ mutant $u b o$ cDNA carrying the S481F and H564R missense mutations. GFPexpressing muscle cell clones are indicated (arrows). (h) Induction of slow-twitch fibers in a smu mutant on ectopic expression of the wild-type ubo cDNA engineered to replace Ser481 with phenylalanine. Slow fibers colabeled with GFP are indicated (arrows). (i) A smu embryo ectopically expressing the wild-type ubo cDNA mutated to replace His564 with arginine, showing a marked reduction in the numbers of slow-twitch fibers induced (long arrows). Note the high proportion of GFP-expressing muscle clones that do not express slow MyHC or Prox 1 (short arrows). Dorsal views are shown in $\mathbf{a}$ and $\mathbf{b}$, lateral views in $\mathbf{c}-\mathbf{i}$. In all panels, embryos are oriented with anterior to the left.

standard methods. An 88-kb PAC clone (BUSMP706F2291), identified by the marker $z 6365$, rescued the $u b o$ phenotype.

The end sequence generated from this clone showed matches to a fully sequenced BAC clone, zK11F5, from the ongoing Danio rerio Sequencing Project. Using genetic markers designed from this sequence, we narrowed the critical genomic interval containing $u b o$ to a region containing one candidate gene, $u b o$, encoding the zebrafish homolog of mammalian Blimp-1. We amplified $u b o$ cDNA from wild-type and pooled $u b o$ embryo RNA and compared several sequences. We used $5^{\prime}$ rapid amplification of DNA ends (Clontech) to identify the first exon of the $u b o$ transcript.

Ubo morpholino oligonucleotides. The sequence of the $u b o$ splice site-targeting morpholino oligonucleotide, designed to inhibit splicing at the intron 2 splice donor junction (Fig. 1f), was 5'-TGGTGTCATACCTCTTTGGAGTCTG$3^{\prime}$. We injected approximately $5 \mathrm{nl}$ of $0.5 \mathrm{mM}$ morpholino oligonucleotide and $5 \mathrm{nl}$ of $0.33 \mathrm{mM}$ morpholino oligonucleotide for the high and low doses, respectively. Only this splice site-specific morpholino oligonucleotide generated morphant embryos that phenotypically resembled $u b o$ mutants; another morpholino oligonucleotide (5'-GGACGCTCTGGTCCCAGCCACACAT- $3^{\prime}$ ), designed to target the translation start site, did not produce effects when used at identical concentrations. We purchased the morpholino oligonucleotides from Gene Tools.

Embryo manipulations, in situ hybridization and antibody labeling. We injected the $u b o$ morpholino oligonucleotide, DNA constructs and $s h$ mRNA into embryos and carried out in situ hybridization and antibody staining using standard procedures. We used a full-length wild-type $u b o$ cDNA in the PCRIITOPO vector as a template for making digoxigenin-labeled antisense riboprobes. The $m y o D$ probe ${ }^{12}$ and the $s h$ mRNA expression construct ${ }^{5,6}$, as well as monoclonal antibody mAbF59 (refs. 2,4) and human serum to Proxl (refs. 2,29), which crossreact with zebrafish slow MyHC and Proxl, respectively, have been described.
Ectopic expression of $\boldsymbol{u b o}$. Because conventional overexpression of $u b o$ with mRNA injection into newly fertilized eggs affects proper gastrulation (S.R., unpublished data), we cloned the wild-type and the $u b o^{\text {tp } 39}$ mutated cDNA into a vector that allows heat shock-dependant mosaic expression from the zebrafish $h s p 70$ promoter at specific developmental stages (F. Maderspacher, unpublished data). In addition, incorporation of the IRES-gfp element (Clontech) into this vector facilitates the synthesis of a bicistronic message and consequent gratuitous labeling of the overexpressing cell clones with the GFP reporter. We generated S481F and H564R variants of the wild-type cDNA by using a site-directed mutagenesis kit (Stratagene) in accordance with the manufacturer's instructions.

We injected $\sim 2-3 \mathrm{nl}$ of the various $h s$-blimp1-IRES-gfp constructs, at a concentration of $25 \mathrm{ng}^{-1} \mathrm{l}^{-1}$, into embryos derived from $s m u^{\mathrm{b} 641}$ heterozygous parents. The embryos were heat-shocked at $38^{\circ} \mathrm{C}$ for $1 \mathrm{~h}$ at the five-somite stage and processed for antibody labeling at 24 h.p.f. A few hours before fixation, the embryos were heat-shocked a second time to facilitate efficient detection of GFP expression in the cell clones. This second heat shock alone did cause the restoration of slow-twitch fiber development in smu mutants. We note, however, that the translation of GFP from the bicistronic mRNA was not highly efficient in zebrafish embryos. Consequently, not all slow-twitch fibers that developed as a result of misexpressing the wild type or the $S 481 \mathrm{~F}$ variant of $u b o$ appeared positively labeled with large amounts of GFP (Fig. 4). For consistency, somites 4-7 on the left half of injected embryos were scored for the incidence of slow-twitch fibers after overexpression of the various $u b o$ constructs. Quantitative analysis of PAC F2291, morpholino oligonucleotide and the different $h s$-blimp1-IRES-gfp injection experiments are given in Supplementary Table 1 online.

URLs. The Zebrafish Information Network, http://zfin.org/; Danio rerio Sequencing Project, http://www.sanger.ac.uk/Projects/D_rerio/.

GenBank accession number. ubo, AY497217. 
Note: Supplementary information is available on the Nature Genetics website.

\section{ACKNOWLEDGMENTS}

We thank F. Wilson and L. Gleadall for help with fish husbandry; R. Geisler for communicating his linkage analysis of the ubo mutation; C. Nüsslein-Volhard and the Max-Planck Institut für Entwicklungsbiologie for the $u b o$ mutant strain; S. Devoto for the $s m u^{\mathrm{b} 641}$ mutant fish; F. Stockdale and S. Tomarev for antibodies; and S. Humphray and K. Jekosch for help with annotating genomic sequences. S.R. is indebted to S. Y. Tay and B. C. Lee for technical assistance and to F. Maderspacher for the hs-IRES-gfp plasmid. This work was funded by grants from the Wellcome Trust and the Medical Research Council to P.W.I. and the Institute of Molecular and Cell Biology and the Agency for Science, Technology and Research, Singapore, to S.R.

\section{COMPETING INTERESTS STATEMENT}

The authors declare that they have no competing financial interests.

Received 25 September; accepted 24 November 2003

Published online at http://www.nature.com/naturegenetics/

1. Lin, J. et al. Transcriptional co-activator PGC-1 $\alpha$ drives the formation of slow-twitch muscle fibers. Nature 418, 797-801 (2002).

2. Roy, S., Wolff, C. \& Ingham, P. The u-boot mutation identifies a Hedgehog regulated myogenic switch for fiber-type diversification in the zebrafish embryo. Genes Dev. 15, 1563-1576 (2001).

3. Turner, C.J. Mack, D. \& Davis, M. Blimp-1, a novel zinc finger-containing protein that can drive the maturation of $B$ lymphocytes into immunoglobulin-secreting cells. Cell 77, 297-306 (1994).

4. Devoto, S., Melancon, E., Eisen, J. \& Westerfield, M. Identification of separate slow and fast muscle precursor cells in vivo, prior to somite formation. Development 122, 3371-3380 (1996).

5. Currie, P.D. \& Ingham, P.W. Induction of a specific muscle cell type by a Hedgehoglike protein in zebrafish. Nature 382, 452-455 (1996).

6. Blagden, C., Currie, P., Ingham, P. \& Hughes, S. Notochord induction of zebrafish slow muscle is mediated by Sonic hedgehog. Genes Dev. 11, 2163-2175 (1997).

7. Du, S., Devoto, S., Westerfield, M. \& Moon, R. Positive and negative regulation of muscle cell identity by members of the hedgehog and TGF- $\beta$ gene families. J. Cell Biol. 139, 145-156 (1997).

8. Lewis, K. et al. Control of muscle cell-type specification in the zebrafish embryo by Hedgehog signaling. Dev. Biol. 216, 469-480 (1999).

9. Barresi, M., Stickney, H. \& Devoto, S. The zebrafish slow-muscle-omitted gene product is required for Hedgehog signal transduction and the development of slow muscle identity. Development 127, 2189-2199 (2000).

10. Wolff, C., Roy, S. \& Ingham, P. Multiple muscle cell identities induced by distinct levels and timing of Hedgehog activity in the zebrafish embryo. Curr. Biol. 13,
1169-1181 (2003).

11. Hammerschmidt, M., Bitgood, M.J. \& McMahon, A.P. Protein kinase A is a common negative regulator of Hedgehog signaling in the vertebrate embryo. Genes Dev. 10, 647-658 (1996)

12. Weinberg, E.S. et al. Developmental regulation of zebrafish MyoD in wild-type, no tail and spadetail embryos. Development 122, 271-280 (1996).

13. Coutelle, 0 . et al. Hedgehog signaling is required for maintenance of $m y f 5$ and myoD expression and timely terminal differentiation in zebrafish adaxial myogenesis. Dev. Biol. 236, 136-150 (2001).

14. Draper, B., Morcos, P. \& Kimmel, C. Inhibition of zebrafish fgf8 pre-mRNA splicing with morpholino oligos: a quantifiable method for gene knockdown. Genesis $\mathbf{3 0}$, 154-156 (2001).

15. de Souza, F. et al. The zinc finger gene Xblimp 1 controls anterior endomesodermal cell fate in Spemann's organizer. EMBO J. 18, 6062-6072 (1999).

16. Ingham, P. \& McMahon, A. Hedgehog signaling in animal development: paradigms and principles. Genes Dev. 15, 3059-3087 (2001).

17. Keller, A. \& Maniatis, T. Only two of the five zinc fingers of the eukaryotic transcriptional repressor PRDI-BF1 are required for sequence-specific DNA binding. Mol. Cell. Biol. 12, 1940-1949 (1992).

18. Kouzarides, T. Histone methylation in transcriptional control. Curr. Opin. Genet. Dev. 12, 198-209 (2002)

19. Lin, Y., Wong, K. \& Calame, K. Repression of c-myc transcription by Blimp-1, an inducer of terminal B cell differentiation. Science 276, 596-599 (1997).

20. Keller, A. \& Maniatis, T. Identification and characterization of a novel repressor of $\beta$-interferon gene expression. Genes Dev. 5, 868-879 (1991).

21. Piskurich, J. et al. BLIMP-1 mediates extinction of major histocompatibility class II transactivator expression in plasma cells. Nat. Immunol. 1, 526-532 (2000).

22. Ren, B., Chee, K., Kim, T. \& Maniatis, T. PRDI-BF1/Blimp-1 repression is mediated by corepressors of the Groucho family of proteins. Genes Dev. 13, 125-137 (1999).

23. Yu, J., Angelin-Duclos, C., Greenwood, J., Liao, J. \& Calame, K. Transcriptional repression by Blimp-1 (PRDI-BF1) involves recruitment of histone deacetylase. Mol. Cell. Biol. 20, 2592-2603 (2000).

24. Lin, K., Angelin-Duclos, C., Kuo, T. \& Calame, K. Blimp-1-dependent repression of Pax -5 is required for differentiation of $\mathrm{B}$ cells to immunoglobulin M-secreting plasma cells. Mol. Cell. Biol. 22, 4771-4780 (2002).

25. Chang, D., Cattoretti, G. \& Calame, K. The dynamic expression pattern of B lymphocyte induced maturation protein-1 (Blimp-1) during mouse embryonic development. Mech. Dev. 117, 305-309 (2002).

26. Borycki, A.-G., Mendham, L. \& Emerson, C. Jr. Control of somite patterning by Sonic hedgehog and its downstream signal response genes. Development 125, 777-790 (1998).

27. van Eeden, F.J.M. et al. Mutations affecting somite formation and patterning in the zebrafish, Danio rerio. Development 123, 153-164 (1996).

28. Talbot, W. \& Schier, A. Positional cloning of mutated zebrafish genes. Methods Cell Biol. 60, 259-286 (1999).

29. Glasgow, E. \& Tomarev, S.I. Restricted expression of the homeobox gene prox 1 in developing zebrafish. Mech. Dev. 76, 175-178 (1998). 